\title{
Cytosine methylation is a conserved epigenetic feature found throughout the phylum Platyhelminthes
}

Kathrin K Geyer ${ }^{1 \dagger}$, lain W Chalmers ${ }^{1 \dagger}$, Neil MacKintosh ${ }^{1}$, Julie E Hirst ${ }^{1}$, Rory Geoghegan ${ }^{1}$, Mathieu Badets ${ }^{2}$, Peter M Brophy", Klaus Brehm ${ }^{3}$ and Karl F Hoffmann ${ }^{1 *}$

\begin{abstract}
Background: The phylum Platyhelminthes (flatworms) contains an important group of bilaterian organisms responsible for many debilitating and chronic infectious diseases of human and animal populations inhabiting the planet today. In addition to their biomedical and veterinary relevance, some platyhelminths are also frequently used models for understanding tissue regeneration and stem cell biology. Therefore, the molecular (genetic and epigenetic) characteristics that underlie trophic specialism, pathogenicity or developmental maturation are likely to be pivotal in our continued studies of this important metazoan group. Indeed, in contrast to earlier studies that failed to detect evidence of cytosine or adenine methylation in parasitic flatworm taxa, our laboratory has recently defined a critical role for cytosine methylation in Schistosoma mansoni oviposition, egg maturation and ovarian development. Thus, in order to identify whether this epigenetic modification features in other platyhelminth species or is a novelty of S. mansoni, we conducted a study simultaneously surveying for DNA methylation machinery components and DNA methylation marks throughout the phylum using both parasitic and non-parasitic representatives.

Results: Firstly, using both S. mansoni DNA methyltransferase 2 (SmDNMT2) and methyl-CpG binding domain protein (SmMBD) as query sequences, we illustrate that essential DNA methylation machinery components are well conserved throughout the phylum. Secondly, using both molecular (methylation specific amplification polymorphism, MSAP) and immunological (enzyme-linked immunoabsorbent assay, ELISA) methodologies, we demonstrate that representative species (Echinococcus multilocularis, Protopolystoma xenopodis, Schistosoma haematobium, Schistosoma japonicum, Fasciola hepatica and Polycelis nigra) within all four platyhelminth classes (Cestoda, Monogenea, Trematoda and 'Turbellaria') contain methylated cytosines within their genome compartments.

Conclusions: Collectively, these findings provide the first direct evidence for a functionally conserved and enzymatically active DNA methylation system throughout the Platyhelminthes. Defining how this epigenetic feature shapes phenotypic diversity and development within the phylum represents an exciting new area of metazoan biology.
\end{abstract}

Keywords: Platyhelminthes, DNA methylation, Epigenetics, Flatworm, 5-methyl cytosine

\footnotetext{
* Correspondence: krh@aber.ac.uk

${ }^{\dagger}$ Equal contributors

${ }^{1}$ Institute of Biological Environmental and Rural Sciences (IBERS), Aberystwyth University, Penglais Campus, Aberystwyth SY23 3FG, United Kingdom

Full list of author information is available at the end of the article
} 


\section{Background}

The Platyhelminthes (flatworms) contain a diverse group of acoelomate, triploblastic organisms. While debate continues as to the evolutionary relationship of the 100,000 or so extant species within this phylum [1], the general consensus remains that these metazoans consist of four major clades: the monophyletic classes Cestoda (endoparasitic tapeworms), Monogenea (mostly ectoparasitic forms) and Trematoda (endoparasitic flukes) as well as the paraphyletic 'Turbellaria' (mostly free-living species). As tractable examples of development and self-regeneration (e.g. turbellarians and cestodes) or as causative agents of aquaculture, veterinary and biomedically-relevant parasitic diseases (e.g. monogeneans, trematodes and cestodes), the platyhelminths are an intensively studied invertebrate group. These research projects have fuelled numerous 'omics' investigations, which in turn, have yielded genetic information pertinent to our current understanding of platyhelminth development, parasite biology, metazoan evolution and adaptation. However, the likely considerable role of 'epigenetics'-based mechanisms in the regulation of these diverse biological processes has yet to be fully investigated [2].

Epigenetics explains how heritable (and potentially reversible) changes in gene expression, caused by mechanisms that do not alter the underlying genetic code, contribute to phenotypic diversity within individuals of a population. Amongst the four major types of epigenetic processes (DNA methylation, histone modifications, miRNA mediated transcriptional regulation and nuclear gene repositioning) found within eukaryotes, DNA methylation is the most highly studied. DNA methylation affects phenotypic diversity by regulating allelic exclusion [3], gene expression [4], embryogenesis [5] and repetitive element silencing [6]. Metazoan DNA methyltransferases (DNMT1, DNMT2, DNMT3a/3b [7]) catalyse this feature by transferring a methyl group $\left(\mathrm{CH}_{3}\right)$ from S-adenosylmethionine (SAM) to the 5-carbon (C5) position of cytosine in gDNA. These 'epigenetic marks' are subsequently recognised by methyl-CpG binding domain proteins (MeCP2 and MBD1-4) and converted into signals necessary for generating phenotypic diversity [8]. Together, DNMTs and MBDs (complexed with other proteins [9]) comprise the core metazoan DNA methylation system found in both vertebrate and invertebrate species.

Historically, DNA methylation was not thought to occur in the phylum Platyhelminthes. This belief was based on a small number of studies, which used rather imprecise methodologies or only sampled restricted developmental lifecycle stages or species [10-12]. However, by utilising a complementary suite of more sensitive technologies and surveying seven distinct lifecycle stages, Geyer et al. recently challenged this longstanding dogma in epigenetics and clearly demonstrated a functional role for DNA methylation in the developmental biology of S. mansoni [13]. In this current investigation, we extend findings of $S$. mansoni DNA methylation to other representative platyhelminth species. We report, for the first time, that DNA methylation (on cytosine residues) and essential DNA methylation machinery components (DNMTs and MBDs) are indeed present in both parasitic and free-living flatworm species. The evolutionary conservation of this epigenetic mark in the developmental progression of monophyletic- (parasitic Cestoda, Monogenea and Trematoda) and paraphyletic - (freeliving 'Turbellaria') platyhelminths $[1,14]$ that inhabit different ecological niches and employ diverse trophic strategies awaits further investigations.

\section{Results/discussion}

\section{The Platyhelminthes contain conserved DNMT2 DNA methyltransferases}

As a phylum, the Platyhelminthes are predominantly studied due to their disease causing potential [15] and/or their suitability as models for investigating regenerative/stem-cell biology [16-18]. Recent genome sequencing- and functional genomics- efforts have led to rapid advances in our understanding of the molecular processes underpinning platyhelminth development, anthelmintic resistance, phenotypic plasticity and host interactions. However, the considerable role of epigenetic-based mechanisms contributing to these phenomena has yet to be fully investigated [2]. Indeed, only recently has a function for cytosine methylation been demonstrated in the developmental biology of a platyhelminth species (S. mansoni; [13]). Here, a role in schistosome egg production was associated with the transcriptional co-regulation of key schistosome DNA methylation machinery components (SmDNMT2 and SmMBD) that mirrored the detection of genomic 5-methyl cytosine $(5 \mathrm{mC})$. Collectively, these data indicated that schistosomes encode a DNA methylation machinery responsible for epigenetic alterations to genome structure and that this process contains components functionally similar to those described in other eukaryotes [7]. Therefore, to investigate whether DNA methylation is uniquely found within the Schistosoma or, rather, features as a conserved regulator driving genomic/phenotypic diversity within other parasitic or free-living platyhelminths, we conducted an investigation searching for essential epigenetic mediators (SmDNMT2/SmMBD homologs) and corresponding epigenetic marks (cytosine methylation) throughout the phylum.

SmDNMT2 (NCBI accession number HM991456) and SmMBD (NCBI accession number HM991455) amino acid and nucleotide sequences as well as Pfam searches were used to identify platyhelminth DNMT and MBD candidates in a variety of publically 
accessible databases [19-24]. Representative species derived from all four platyhelminth classes (Trematoda S. japonicum, S. haematobium, Fasciola hepatica, Clonorchis sinensis and Opisthorchis viverrini; Monogenea - Neobenedenia melleni; Cestoda - E. multilocularis, Echinococcus granulosus, Taenia solium, Hymenolepis microstoma; Turbellaria - Schmidtea mediterranea and Macrostomum lignano) contained candidates with high degrees of sequence similarity to SmDNMT2, Mus musculus DNMT2 and Schizosaccharomyces pombe DNMT2 (PMT1) (Figure 1). Analysis of the thirteen platyhelminth sequences encoding putative DNMT candidates (together with SmDNMT2, MmDNMT2 and PMT1) indicated that they all lacked the large N-terminal regulatory region found in DNMT1 and DNMT3 enzymes [25] as well as an obvious nuclear localization signal (NLS). In contrast, all but two of the putative platyhelminth DNMTs (N. melleni and O. viverrini being exceptions as they originate from incomplete database entries), contained all ten DNMTcharacteristic motifs (I-X) and the target recognition domain (TRD) arranged in the correct order within the C-terminal catalytic, DNA methyltransferase domain (PF00145) [26,27]. Detailed analysis of the six most highly conserved catalytic domain motifs (I, IV, VI, VIII, IX and X) as well as the TRD indicated high sequence conservation across the candidates (Figure 1). Specifically, the enzymatically important cysteine (C76 in SmDNMT2) within the proline/cysteine dipeptide in motif IV is invariant amongst all platyhelminth DNMT candidates. This cysteine mediates covalent bond formation to the target cytosine and is functionally dependent upon the presence of the proceeding proline (P75 in SmDNMT2) [28]. In contrast to PMT1, which is not a functional DNA methyltransferase due to a $\mathrm{P}$ to $\mathrm{S}$ substitution [29], all platyhelminth DNMT candidates
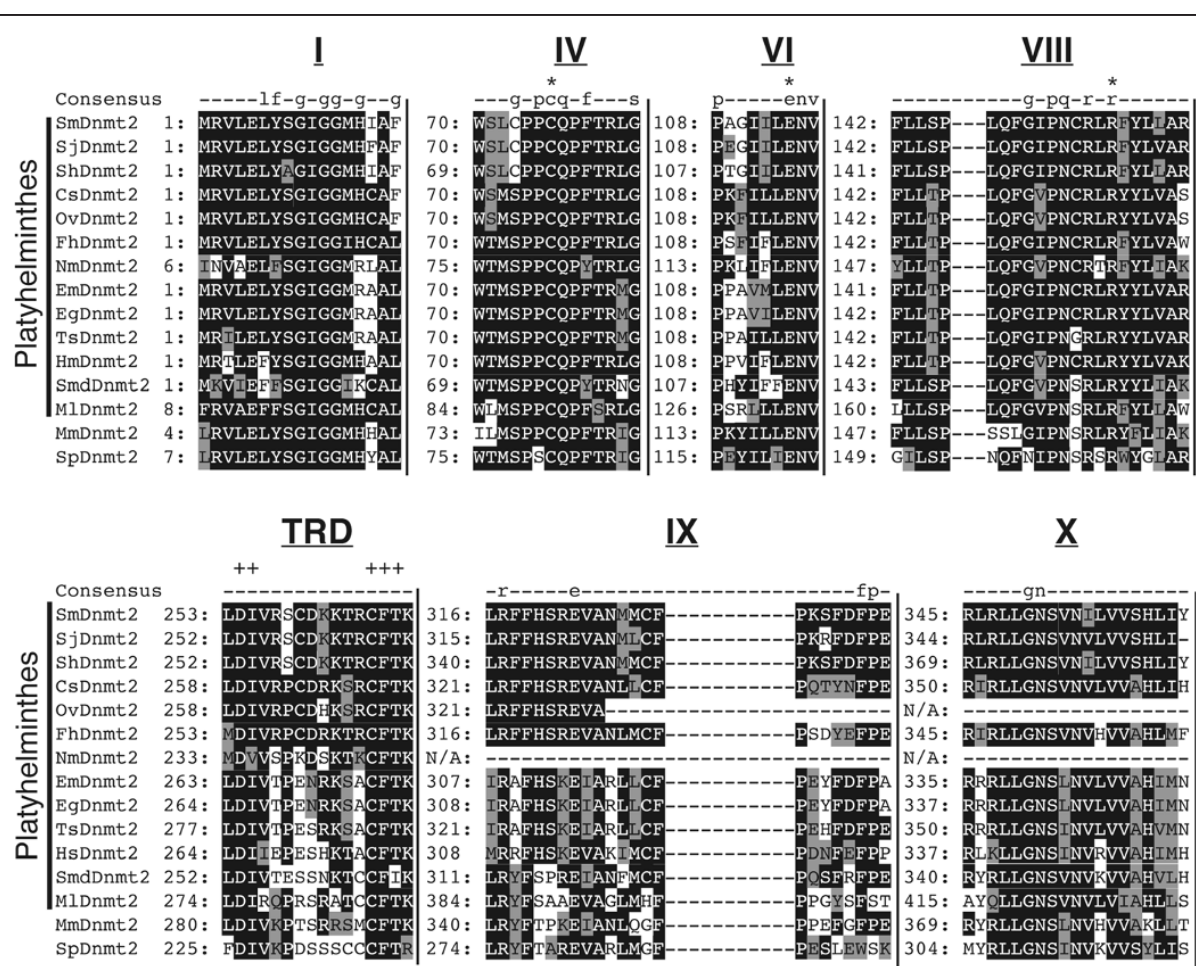

$\underline{\text { IX }}$

$\underline{\mathbf{X}}$

Figure 1 Platyhelminth DNMT candidates share extensive sequence similarity across the six conserved motifs and the target recognition domain (TRD) of DNA methyltransferase enzymes. A concatenated multiple sequence alignment of DNMT candidates from S. mansoni (Sm), S. japonicum (Sj), S. haematobium (Sh), C. sinensis (CS), O. viverrini (Ov), F. hepatica (Fh) N. melleni (Nm), E. multilocularis (Em), E. granulosus (Eg), T. solium (Ts), H. microstoma (Hm), S. mediterranea (Smd) and M. lignano (MI) with M. musculus DNMT2 and S. pombe PMT1 (NCBI accession numbers are listed in the Methods). The six highly conserved motifs in this catalytic domain are indicated above the alignment in Roman numerals (I, IV, VI, VIII, IX and X) as is the TRD. Numbers at the beginning of each motif represent amino acid positions and at each position the most conserved residues are further shaded in black, semi-conserved residues are highlighted grey and non-conserved amino acids are kept white. The row labelled 'consensus' represents the Pfam consensus sequence of the DNA methyltransferase (PF00145) domain where conserved amino acid residues (50-79\%) are indicated by lower-case letters and highly conserved residues (> 80\%) are indicated by upper-case letters. Functionally important cysteine (C), glutamic acid (E) and arginine (R) residues within motifs IV, VI and VIII respectively, are indicated by an asterisk $\left(^{*}\right)$ above the 'consensus' row. DNMT2 specific residues within the target recognition domain (TRD) are indicated by a plus ( + ) above the 'consensus' row. Missing amino acid residues, not present in truncated OvDNMT and NmDNMT candidates are indicated by a 'N/A'. 
(as well as MmDNMT2) contain this structurally relevant proline residue.

Another invariant region found amongst the platyhelminth DNMT candidates is the glutamic acid/asparagine/valine tripeptide (E114/N115/V116 in SmDNMT2) of motif VI (Figure 1). Within this tripeptide, the glutamic acid residue utilises hydrogen bonding to interact with the target cytosine and is necessary for the stability of the enzyme/target complex [28]. The motif VIII arginine (R157 in SmDNMT2) also facilitates hydrogen bonding to stabilise enzyme/cytosine complexes [28] and is, similar to SmDNMT2 E114, absolutely conserved across the representative platyhelminth DNMTs (Figure 1).

The presence of these six motifs (I, IV, VI, VIII, IX and $\mathrm{X}$ ) within the C-terminal catalytic domain (PF00145) and the absence of DNMT1 and DNMT3 N-terminal regulatory domains strongly indicate that these new platyhelminth DNMTs are SmDNMT2 homologs. Additionally, conserved aspartic acid/isoleucine dipeptides (D254/I255 in SmDNMT2) and cysteine/phenylalanine/ threonine tripeptides (C265/F266/T267 in SmDNMT2) within the TRD (between motifs VIII and IX) add further support to this assertion (Figure 1). These two polypeptide regions are specific to DNMT2 family members and are not found in DNMT1, DNMT3 or bacterial DNA methyltransferases. Importantly, the CFT tripeptide has been additionally proposed to help coordinate target recognition during enzyme interactions [27]. Therefore, strong CFT conservation in all but one (S. mediterranea contains a T266 to I266 substitution in the CFT tripeptide) platyhelminth DNMT candidate further supports their inclusion as new DNMT2 family members. While vertebrate DNMT2 family members are now considered predominant tRNA methyltransferases [30], many invertebrate/ single-cell eukaryote DNMT2s still retain strong DNA methyltransferase activity [31-34]. The enigmatic role and genomic targets of these new platyhelminth DNMT2s await enzymatic and functional characterisation.

Phylogenetic reconstruction of the platyhelminth DNMT2 family with other characterised DNMTs (e.g. M. musculus, Ciona intestinalis and Apis mellifera DNMT1, DNMT2, DNMT3 members) reveals that, despite minor sequence differences in the platyhelminth homologs, they all cluster in a monophyletic clade (Figure 2, dashed box). These platyhelminth DNMT2 homologs are additionally found within a larger clade that contains MmDNMT2 (mouse), CiDNMT2 (sea squirt), AmDNMT2 (honey bee), CqDnmt2 (mosquito), HrDNMT2 (leech), CtDNMT2 (polychaete annelid), LgDNMT2 (limpet) and CgDNMT2 (oyster) members (Bayesian posterior probability support value = 1; Maximum Likelihood bootstrap value = 97\%) adding further evidence for their inclusion as novel DNMT2, but not DNMT1 or DNMT3, DNA methyltransferases. The large genetic distances between DNMT2 family members is likely attributed to their presumed dual substrate (DNA and RNA) specificities [35] when compared to DNMT1 or DNMT3 exemplars (only harbouring DNA methyltransferase activity). Therefore, enzymatic analyses of the platyhelminth DNMT2s remain an important priority to provide a mechanistic explanation for their greater evolutionary divergence when compared to DNMT1 and DNMT3 family members.

\section{The Platyhelminthes contain conserved MBD2/3 methyl- CpG binding proteins}

Similar to the newly identified platyhelminth DNMT2 homologs, mostly intact MBD candidates were found in the Trematoda (S. japonicum, F. hepatica, Paragonimus westermani, C. sinensis and O. viverrini), the 'Turbellaria' (S. mediterranea and M. lignano) and the 'Cestoda' (E. multilocularis, E. granulosus, T. solium and $H$. microstoma). The exception to our analysis was the 'Monogenea', where no MBD candidate was found. It is likely that increased numbers of platyhelminth MBDs (especially within the 'Monogenea') will be identified once existing genomes undergo revision [36] or new genomes/ transcriptomes are sequenced. Nonetheless, multiple sequence alignment of these platyhelminth MBD candidates with SmMBD, MmMBD2 (mouse) and MmMBD3 (mouse) demonstrated conservation over the 70aa, Nterminal methyl-CpG binding domain (PF01429) and the 97aa, C-terminal domain of methyl-CpG binding protein 2 and 3 (PF14048) (Figure 3). The conservation of these two domains and the fact that only one MBD candidate was found in each of the platyhelminth genomes/ transcriptomes suggested that these proteins (in addition to SmMBD) are new members of the ancestral MBD2/3 family [37]. This contention is further supported by the lack of both MBD1 characteristic CxxC Zn-finger motifs [38] and MBD4 characteristic glycosylase DNA repair domains [39]. Furthermore, phylogenetic reconstruction of these novel platyhelminth MBD homologs places them within a large clade (Bayesian posterior probability support value $=0.58$; Maximum Likelihood bootstrap value $=$ 45\%) containing MBD2/3 (Hemicentrotus pulcherrimus Bombyx mori, Capitella teleta, Crassostrea gigas, Lottia gigantea and Helobdella robusta MBD2/3s) proteins, and the vertebrate MBD2 and MBD3 homologs (M. musculus and Xenopus laevis) but outside a distinct clade containing MeCP2 (X. laevis and M. musculus MeCP2s), MBD1 (X. laevis and M. musculus MBD1s) and MBD4 (X. tropicalis and $M$. musculus MBD4s) exemplars (Figure 4). Collectively, these results strongly support the platyhelminth MBDs as being novel members of the invertebrate specific MBD2/3 family. 


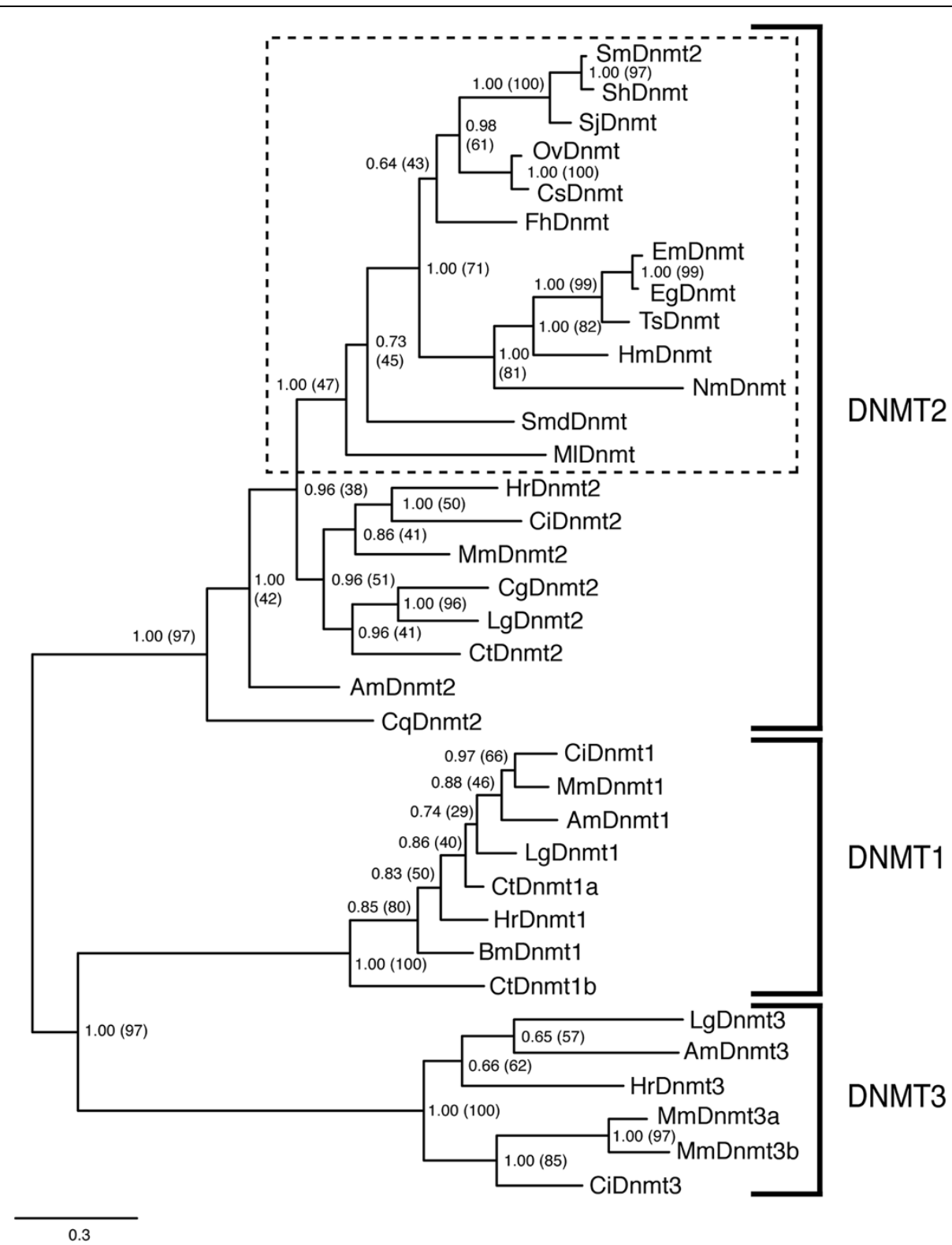

Figure 2 Platyhelminth DNMTs are novel monophyletic members of the DNMT2 DNA methyltransferase family. Phylogenetic relationships were inferred from a concatenated multiple sequence alignment (MUSCLE v3.8) of platyhelminth and representative eukaryotic DNMT sequences (NCBI accession numbers are listed in Methods), using Bayesian (implemented in MrBayes v3.1.2) and Maximum Likelihood (implemented in MEGA v5.05) approaches. The figure shows the Bayesian consensus tree illustrated by TreeView, where branch lengths (indicated by scale bar) represent distance between taxa. Only nodes with Bayesian posterior probability support values greater than 0.5 are shown. Node labels within parentheses represent percentage bootstrap support values from Maximum Likelihood analysis (1000 bootstrap replicates performed), while those outside parentheses represent Bayesian posterior probability support values. Platyhelminth sequence names are as listed in Figure 1. Notations Am, Mm, Bm, $\mathrm{Ci}, \mathrm{Cq}, \mathrm{Ct}, \mathrm{Cg}$, Lg and Hr correspond to A. mellifera, M. musculus, B. mori, C. intestinalis, C. quinquefasciatus, C. teleta, C. gigas, L. gigantea and H. robusta sequences respectively. The platyhelminth DNMT2 clade is indicated by a dashed box.

Despite sharing greater than $70 \%$ amino acid identity, indicative of a gene duplication event [37], mammalian MBD2 is capable of binding methylated cytosine within gDNA whereas MBD3 is not [40]. Therefore, identifying whether these new platyhelminth MBD2/3 proteins share greater sequence similarity to methyl-CpG binding MBD2 or non methyl-CpG binding MBD3 members was investigated. Specific attention was focused on amino acids known to be important for MBD function (i.e. binding to methyl-CpG) and included the following residues: arginine 58 (R58), lysine 66 (K66), tyrosine 79 (Y70) and arginine 80 (R80) (SmMBD2/3 numbering used unless otherwise stated; Figure 3, asterisks above PF01429 consensus sequence). Both $\mathrm{K}$ and Y residues 


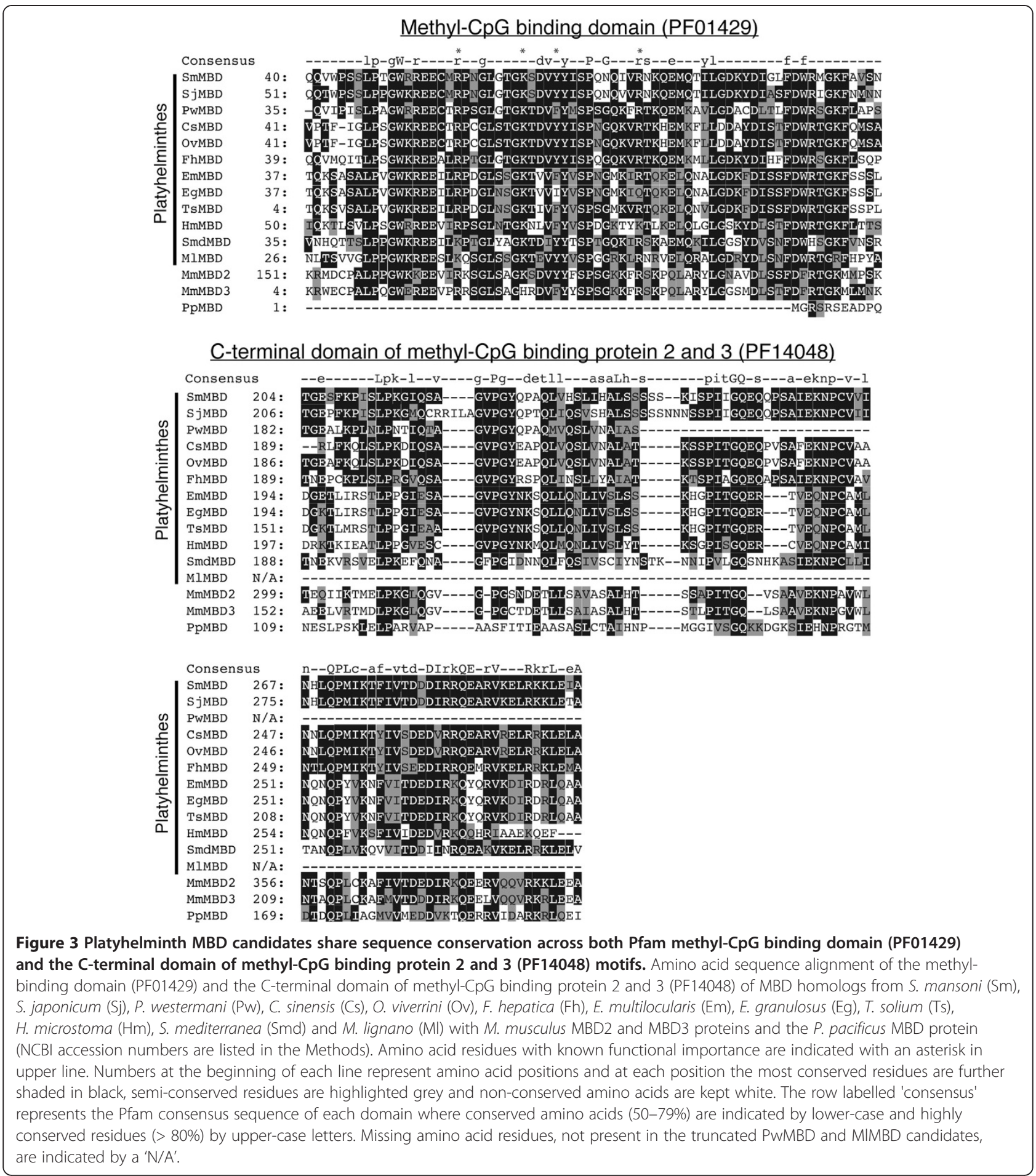

are important for MBD function (e.g. Y residue coordinating $\mathrm{H}$-bonding to $5 \mathrm{mC}$ [41]) as mammalian MBD3 contains $\mathrm{K} 30$ to $\mathrm{H} 30$ and $\mathrm{Y} 34$ to F34 substitutions (Figure 3) and is unable to bind methylated cytosine [40]. In addition, both $\mathrm{R}$ residues also are critical in $\mathrm{MBD} /$ target $(5 \mathrm{mC})$ interactions as demonstrated by recent X-ray crystallography studies $[42,43]$. Whereas all platyhelminth MBD2/3s contain conserved K66 and $\mathrm{R} 80$ residues, there is variability in the conservation of SmMBD2/3 Y70 and R58 residues. For example, the Y70 amino acid position is maintained in SmMBD2/3, SjMBD2/3, CsMBD2/3 and OvMBD2/3, but is substituted for an $\mathrm{F}$ in $P$. westermani MBD2/3 and all of the cestode sequences (E. multilocularis, $T$. solium, $H$. microstoma 


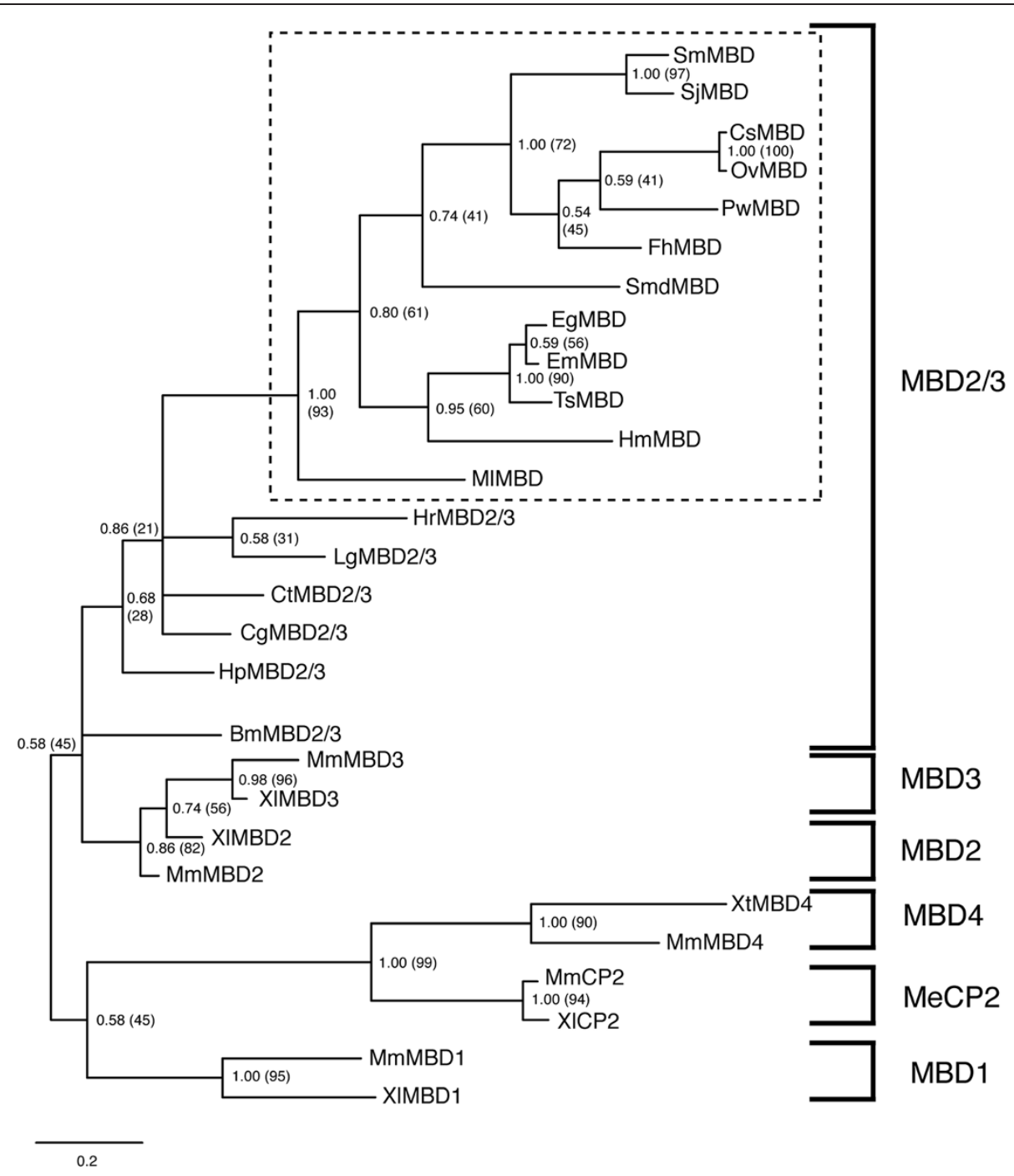

Figure 4 Platyhelminth MBD candidates are novel members of the invertebrate specific MBD2/3 clade of methyl-CpG binding domain containing proteins. Phylogenetic relationships were inferred from a multiple sequence alignment (MUSCLE v3.8) of platyhelminth and representative eukaryotic MBD domains (NCBI accession numbers are listed in the Methods) using Bayesian (implemented in MrBayes v3.1.2) and Maximum Likelihood (implemented in MEGA v5.05) approaches. The figure shows the Bayesian consensus tree illustrated by TreeView, where branch lengths (indicated by scale bar) represent distance between taxa; only nodes with Bayesian posterior probability support values greater than 0.5 are shown. Node labels within parentheses represent percentage bootstrap support values from Maximum Likelihood analysis (1000 bootstrap replicates performed), while those outside represent Bayesian posterior probability support values. Notations Hp, Bm, XI, Xt, Mm, $\mathrm{Ct}, \mathrm{Cg}$, Lg and $\mathrm{Hr}$ correspond to H. pulcherrimus, B. mori, X. laevis, X. tropicalis, M. musculus, C. teleta, C. gigas, L. gigantea and H. robusta sequences. The platyhelminth MBD2/3 clade is indicated by a dashed box.

and E. granulosus). Furthermore, in $S$. mediterranea SmdMBD2/3, M. lignano MlMBD2/3 and $H$. microstoma HmMBD2/3 sequences, a $\mathrm{R}$ to $\mathrm{K}$ substitution at position 58 is also observed (Figure 3). Based on structural and comparative studies, both of these substitutions likely affect the methyl-CpG binding ability of $P$. westermani, $S$. mediterranea $M$. lignano and cestode MBD2/3 proteins [41] and may indicate additional/altered functions of these platyhelminth members (perhaps similar to MBD3L1/MBD3L2 [44], MBD3 [45] or other MBD2/3 $[46,47]$ homologs). For SmMBD2/3, SjMBD2/3, CsMBD2/3 and OvMBD2/3 members, containing the full repertoire of functionally-conserved amino acid residues in their methyl-CpG binding domains, it is likely that these proteins bind $5 \mathrm{mC}$. However, as shown for Drosophila melanogaster MBD2/3 [48], $5 \mathrm{mC}$ recognition of these platyhelminth members may be predominantly in the $\mathrm{CpT} / \mathrm{CpA}$ context. Evidence to support this contention was recently provided in $S$. mansoni, where cytosine methylation was predominantly found in the CpA context $[12,13]$. Clearly, further investigations are necessary to extend this locus-specific observation and identify the 
repertoire of activities mediated by platyhelminth MBD2/3 proteins.

\section{DNA methylation is found in all four platyhelminth classes}

Having established that representative species across all four classes within the Platyhelminthes contain a SmDNMT2 homolog (Figures 1 and 2), we next investigated whether DNA methylation was also conserved across the phylum (Figure 5). Previously, using a variety of technologies including methylation sensitive amplification polymorphism (MSAP), cytosine methylation was found in the $S$. mansoni genome and this epigenetic feature was directly dependent upon the presence of enzymatically active SmDNMT2 [13]. Therefore, the genomes of related schistosome species, S. japonicum (containing SjDNMT2) and S. haematobium (containing ShDNMT2), were first examined for cytosine methylation (in the $\mathrm{CpG}$ context) using the MSAP technique (Figure 5A). Here, principal coordinate analyses (PcoA) of both S. japonicum and S. haematobium mixed-sex adult parasite gDNA samples, differentially digested with MspI and HpaII isoschizomers, clearly indicated the presence of genomic loci containing $5 \mathrm{mC}$. This finding confirmed that all three medically important schistosome species contained a methylated genome and that MSAP was sensitive enough to detect this epigenetic modification in mixed-sex adult gDNA samples. Similar to $S$. mansoni, it is likely that $5 \mathrm{mC}$ detected in mixedsex adult S. japonicum and S. haematobium samples is preferentially associated with females where this DNA modification system has previously been shown to regulate oviposition [13]. If this is the case, then selective targeting of Schistosoma DNMT2s may represent a pangenus control strategy useful for novel schistosomiasis interventions.

We next used MSAP to identify cytosine methylation in E. multilocularis (containing EmDNMT2, Figures 1 and 2) protoscoleces and P. xenopodis (monogenean parasite related to NmDNMT2-containing N. mellini, Figures 1 and 2) adults obtained from the urinary bladder of infected $X$. laevis (Figure 5A). Discrete segregation (between $M s p \mathrm{I}$ and HpaII digested populations) along the first principal coordinate indicated clusters of genomic loci containing this epigenetic modification in both parasitic platyhelminths. Importantly, this is the first molecular evidence for global cytosine methylation in the genomes of both Cestoda and Monogenea species. In contrast to $E$. multilocularis and $P$. xenopodis genomes, we were unable to confidently extend MSAP to detect cytosine methylation in F. hepatica (hermaphroditic trematode related to dioecious schistosomes) and P. nigra (turbellarian related to SmdDNMT2-containing S. mediterranea or MIDNMT2-containing M. lignano,
Figures 1 and 2) genomes (collected from individual worms). Despite the suggestion of MSAP-detectable DNA methylation in these two species (HpaII and MspI populations are generally separated, Additional file 1), the presence of genetic diversity amongst sampled replicates averts the inference of epigenetic distance. The genetic diversity manifested itself in parasite MspI (methylation insensitive enzyme; indicative of genomic homozygosity/heterozygosity) populations contributing to more variability than parasite HpaII (methylation sensitive enzyme; indicative of methylated genomic loci) populations during multivariate analysis (Additional file 1). The environmental sources of both $F$. hepatica (sheep from different provenances) and P. nigra (rocky shores of Llyn-yr-Oerfa) led to sampling of outbred individuals and likely accounted for this genetic diversity.

Therefore, to survey for $5 \mathrm{mC}$ in these particular platyhelminth species, an alternative method for detecting $5 \mathrm{mC}$ was employed (Figure 5B). This involved an ELISA-based approach, which also was used to successfully identify $5 \mathrm{mC}$ in S. mansoni [13] and M. musculus [49]. Using this methodology, $5 \mathrm{mC}$ was found in both E. multilocularis and $P$. xenopodis genomes (Figure 5B), confirming the MSAP results for these parasites (Figure 5A) and providing confidence that ELISAbased detection of this genome modification could be translated from Schistosoma to other platyhelminth genomes. When subsequently applied to $P$. nigra and $F$. hepatica genomes, clear evidence for $5 \mathrm{mC}$ was obtained (Figure 5B). While not exhaustive, this ELISA-based study suggested that digenean trematodes ( $F$. hepatica) contain higher levels of $5 \mathrm{mC}$ when compared to representative cestode- (E. multilocularis), monogenean( $P$. xenopodis) and turbellarian- ( $P$. nigra) species. Quantitative detection of $5 \mathrm{mC}$ in additional platyhelminth species (across the four classes within the phylum) would help to clarify this observation.

\section{Conclusions}

The combined presence of conserved DNMT2 and MBD2/3 candidates, as well as cytosine methylation marks throughout the Platyhelminthes, suggests that epigenetic-mediated DNA methylation features in the complex biology of this important phylum. However, it remains to be determined if all species within this phylum have maintained this epigenetic trait or, similar to the Arthropoda [34,50-54] and Nematoda [55], some platyhelminths (especially within the paraphyletic 'Turbellaria' [14]) have lost this inheritable mechanism for generating phenotypic diversity. Furthermore, identifying the full enzymatic and target specificities of platyhelminth DNMT2 homologs requires additional investigation. It is currently unknown whether platyhelminth DNMT2s, in addition to DNA, have an 

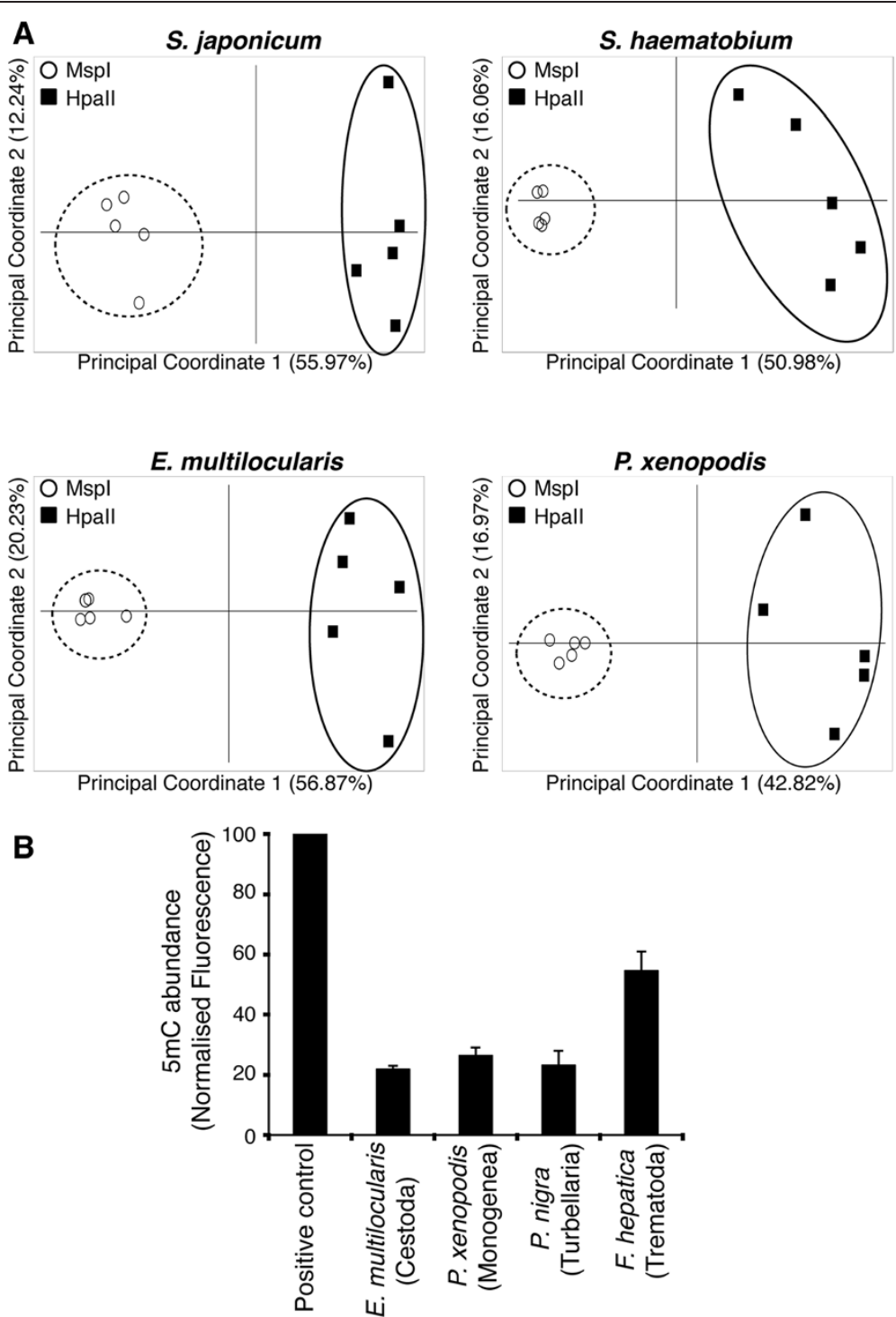

Figure 5 Global DNA methylation is found in representative species across all four Platyhelminthes classes. A) MSAP analysis of $5 \mathrm{mC}$ in gDNA obtained from S. japonicum mixed-sex adults, S. haematobium mixed-sex adults, E. multilocularis protoscoleces and P. xenopodis adults. Hpall (filled squares) and Mspl (open circles) isoschizomers were used to restrict gDNA samples. Five replicate samples were analysed for all platyhelminth gDNA samples (biological replicates for mixed-sex adult schistosome populations, biological replicates for adult $P$. xenopodis individuals and technical replicates for E. multilocularis protoscoleces). MSAP data ( $\%=$ the two components explaining the most variability within the dataset) is illustrated by principal coordinate analysis (Mspl replicates enclosed by dashed circle; Hpall replicates enclosed by solid oval). B) ELISA detection of $\mathrm{m} 5 \mathrm{C}$ in E. multilocularis protoscoleces, $P$. xenopodis adults, P. nigra adults and F. hepatica adults. Histograms (+SD, standard deviation) represent mean $\mathrm{m} 5 \mathrm{C}$ abundance ( $\mathrm{n}=3$ for each platyhelminth gDNA sample), which was normalised (to both positive and negative control DNA samples) according to the Methods. Data is representative of two independent experiments.

affinity for tRNA substrates [30]. Also, the only study to investigate DNA methylation at single base pair resolution within the Platyhelminthes has produced ambiguous results likely due to sampling of $S$. mansoni adult male gDNA only [12]. In previous studies this schistosome developmental form has been shown to contain the lowest level of cytosine methylation throughout the parasite's lifecycle [13]. Future work will help characterise additional roles of platyhelminth DNMT2s and lead to the identification of genomic positions (e.g. repetitive elements, coding/non-coding genomic loci) targeted by this core DNA methylation machinery component. By doing so in a comprehensive manner (elucidation of DNA methylomes from various lifecycle stages and different platyhelminth species), insights into regenerative-, stem cell- and developmental-biology will be obtained leading to both a greater understanding of the complex life 
histories within the Platyhelminthes and novel opportunities for control.

\section{Methods}

\section{Ethical approval}

Animal handling, care and subsequent experimentation were performed in compliance with German (Deutsches Tierschutzgesetz,TierSchG, version from Dec-9-2010) and European (European directive 2010/63/EU) regulations on the protection of animals. Ethical approval of the study was obtained from the local ethics committee of the government of Lower Franconia (Regierung von Unterfranken 55.2-2531.01-31/10).

\section{Platyhelminth material and gDNA isolation}

Mixed sex, adult S. japonicum and S. haematobium worms (blood flukes, class Trematoda) were obtained from perfusion of infected mice and hamsters, respectively [56]. Adult $F$. hepatica worms (liver flukes, class Trematoda) were carefully removed from livers and bile ducts of naturally infected sheep (collected from Randell Parker Foods, Llanidloes, Wales). These parasites were washed six times in pre-warmed $\left(37^{\circ} \mathrm{C}\right)$ phosphate buffered saline (PBS, pH 7.3) and cultured (one adult/well in a six well tissue culture plate) in DMEM (Sigma-Aldrich, UK) supplemented with $10 \%$ foetal calf serum, $2 \mathrm{mM}$ $L$-glutamine, $100 \mathrm{U} / \mathrm{ml}$ penicillin and $100 \mu \mathrm{g} / \mathrm{ml}$ streptomycin in an atmosphere of $5 \% \mathrm{CO}_{2}$ for $48 \mathrm{hr}$ with a $70 \%$ media exchange performed after $24 \mathrm{hr}$. P. xenopodis (class Monogenea) adult worms were collected from urinary bladders of infected X. laevis originating in Potchefstroom, South Africa. P. nigra (class Turbellaria) were collected from stones at the edges of Llyn-yr-Oerfa (Mid Wales) and maintained in a cold-water aquarium for 6 days without food. JAVA, a natural E. multilocularis isolate from the liver of an infected cynomolgus monkey (Macaca fascicularis; [57]) was passaged intraperitoneally through laboratory jirds (Meriones unguiculatus; [58]). E. multilocularis genomic DNA (gDNA) was isolated from a pure protoscolex preparation [59] after treatment with pepsin at $\mathrm{pH} 2$ [60] according to a previously established protocol [61]. The remaining platyhelminth gDNA samples were extracted using the Qiagen DNeasy Kit. All gDNA samples were subsequently quantified by a Nanodrop-1000 spectrophotometer. Where possible ( $S$. japonicum, F. hepatica, P. xenopodis and E. multilocularis), gDNA was subjected to host-contamination checks by PCR as previously described [13,62] (Additional file 2).

\section{Platyhelminth DNMT2 and MBD sequence identification}

DNMT2 and MBD members from platyhelminth species were identified by tBLASTn searches of transcripts, predicted genes and genome assemblies of NCBI [19], Welcome Trust Sanger Institute [63] and Gasser
Laboratory [20] EST databases as well as T. solium [22], S. mediterranea (SmedGD v1.3.14; [21]), M. lignano [64] and $F$. hepatica [65] gene models/genomic databases using SmDnmt2 IsoformI (NCBI accession no. HM991456) and SmMBD (NCBI accession no. HM991455) as query sequences. A Pfam domain search on SchistoDB v3.0 (beta version; [23]) was performed to identify a DNMT homolog within the genome of S. haematobium. Searches were performed on 25-01 -12 for S. japonicum, S. haematobium, P. westermani, C. sinensis, O. viverrini, N. melleni and S. mediterranea, 14-11-12 for F. hepatica and M. lignano and 01-05-13 for E. granulosus, E. multilocularis, T. solium and $H$. microstoma. Where multiple ESTs (or ESTs and gene predictions) existed of the same homolog, a consensus sequence was formed. In the cases of $S$. haematobium, M. lignano, F. hepatica, E. multilocularis, E. granulosus, $H$. microstoma and $T$. solium DNMT homologs, the exon-intron structure of SmDNMT2 IsoformI was used as a reference to identify/correct the platyhelminth DNMT prediction. All exon/intron junctions in these altered gene predictions conformed to the consensus (GT/AG) splice donor/acceptor sequences for eukaryotes. Final amino acid sequences used in the study are listed in Additional file 3.

\section{Sequence alignments and phylogenetic trees}

Multiple sequence alignments of platyhelminth DNMT and MBD candidates were generated using MUSCLE v3.8 [66]. Domain predictions were performed using the Pfam web server [67] to define the limits of the C-5 cytosine-specific DNA methylase domain (DNMT; PF00145), Methyl-CpG binding domain (MBD; PF01429) and $\mathrm{C}$-terminal domain of methyl-CpG binding protein 2 and 3 (MBD_C; PF14048) regions for each sequence. Motif positions represented in the DNMT multiple sequence alignment were derived from a previous report [68]. In addition to the identified platyhelminth candidates, the following sequences were included in the multiple sequence alignments as representative DNMT and MBD members: M. musculus MBD2 (NM_010 773), M. musculus MBD3 (NM_013595), Pristionchus pacificus MBD2/3 (AAV85979.1), M. musculus DNMT2 (AAC53529.1) and S. pombe DNMT2 (CAA57824.1).

For phylogenetic analysis (using Maximum Likelihood and Bayesian approaches) of platyhelminth and nonplatyhelminth DNMT members, amino acid sequences were aligned using MUSCLE software with minor improvements in the alignment performed following visual inspection. Non-conserved regions were then removed from the alignment to allow for phylogenetic comparison (during this step the target recognition domain (TRD) and variable regions were removed). In total, 230 amino acid positions were used in the analyses. The 
non-platyhelminth sequences (and their NCBI accession numbers) included in the DNMT phylogenetic analyses were as follows: $M$. musculus DNMT1 (P13864.5), M. musculus DNMT2 (AAC53529.1), M. musculus DNMT3a (O88508.2), M. musculus DNMT3b (O88509.2), A. mellifera DNMT1 (NP_001164522.1), A. mellifera DNMT2 (XP_393991.3), A. mellifera DNMT3 (NP_001177350.1), C. intestinalis DNMT2 (XP_002128135.1), C. intestinalis DNMT1 (XP_00212 2948.1), C. intestinalis DNMT3a (XP_002123461.1), Culex quinquefasciatus DNMT2 (XP_001867327.1), B. mori DNMT1 (NP_001036980.1), C. teleta Dnmt1a (ELT93682.1), C. teleta Dnmt1b (ELU12454.1), C. teleta Dnmt2 (ELU13416.1), C. gigas Dnmt2 (EKC25 033.1), L. gigantea Dnmt1 (transcript name: 114987 [69]) L. gigantea Dnmt2 (transcript name: 119453 [69]), L. gigantea Dnmt3 (transcript name: 171288 [69]), $H$. robusta (transcript name: 116156 [69]), H. robusta Dnmt2 (transcript name: 89038 [69]) and H. robusta Dnmt3 (transcript name: 162653 [69]). The Bayesian phylogenetic analyses were performed as described previously [70] using MrBayes v3.1.2 [71], the WAG substitution model [72] and a minimum of 1 million generations. The log-likelihood score of each saved tree was plotted against the number of generations to determine the point at which the log-likelihood scores of the analyses stabilised. Maximum Likelihood analysis was conducted using MEGA 5.0 [73] with the WAG substitution model and 1000 bootstrap replicates. The graphical output of the final Bayesian consensus phylogram was obtained using Figtree v1.3.1 [74]. This tree was then exported into Adobe Illustrator where Maximum Likelihood bootstrap support values for each node were superimposed.

Phylogenetic analyses of the MBD proteins were performed using a MUSCLE alignment restricted to the MBD domain regions of the sequences (as defined by Pfam; PF01429). The non-platyhelminth sequences (and their NCBI accession numbers) included in the MBD phylogenetic analyses were as follows: M. musculus MBD1 (NM_013594), M. musculus MBD2 (NM_010 773), M. musculus MBD3 (NM_013595), M. musculus MBD4 (NM_010774), M. musculus MeCP2 (NM_010 788), X. laevis MBD1 (NP_001104183.1), X. laevis MBD2 (NP_001083787.1), $\quad \bar{X}$. laevis MBD3 (BAC 22082.1), X. tropicalis MBD4 (NP_001037916.1), X. laevis MeCP2 (AAD03736.1), H. pulcherrimus MBD2/3 (EU590662), B. mori MBD2/3 (BGIBMGA001425-PA; SilkDB [75]), C. teleta MBD2/3 (ELT95247.1), C. gigas MBD2/3 (EKC32831.1), L. gigantea MBD2/3 (transcript name: 112523 [69]) and $H$. robusta MBD2/3 (transcript name: 185546 [69]). Phylogenetic relationships between the 20 members were then inferred in the same way as for the DNMT homologs, using Maximum Likelihood and Bayesian approaches. Maximum Likelihood bootstrap support values for each node (in parentheses) were superimposed onto the Bayesian consensus phylogram using Adobe Illustrator.

\section{Methylation specific amplification polymorphism (MSAP) analysis of platyhelminth $\mathrm{CpG}$ methylation}

The MSAP method [76], modified for the detection of CpG methylation in S. mansoni [13], was applied to platyhelminth gDNA samples free from host contamination (Additional file 2). Five biological replicates were analysed for $P$. xenopodis (single adults), F. hepatica (single adults), $P$. nigra (single adults), S. haematobium (mixed-sex adults) and S. japonicum (mixed-sex adults) samples. Five technical replicates were examined for E. multilocularis protoscolex samples. Following amplicon size determination on an ABI 3100 DNA capillary sequencer (IBERS, Aberystwyth), the raw data was viewed and analysed in GeneMapper v4.0. GenAlex v6.4 was subsequently employed for multivariate Principal Coordinate Analysis (PcoA).

\section{ELISA detection of $5 \mathrm{mC}$}

The amount of $5 \mathrm{mC}$ in $100 \mathrm{ng}$ of selected platyhelminth gDNA samples was analysed using the SuperSense Methylated DNA Quantification Kit (Epigentek) as previously described [13]. Reactions were carried out in triplicates for each platyhelminth sample (P. nigra, $F$. hepatica, P. xenopodis and E. multilocularis) and in duplicates for the negative and positive control (both supplied with the kit). Fluorescent readings $\left(530_{\mathrm{ex}} /\right.$ $590_{\mathrm{em}} \mathrm{nm}$ ) were obtained using a POLARstar Omega (BMG Labtech, UK) microtiter plate reader and samples were subsequently normalised to controls. The mean of the fluorescent readings obtained from the negative control sample was subtracted from the platyhelminth and positive control values. Subsequently, the fluorescent mean of the positive control values was set at $100 \%$ $(5 \mathrm{mC}=100 \%)$, and the $5 \mathrm{mC}$ abundance in each platyhelminth sample was normalised to this value.

\section{Additional files}

Additional file 1: MSAP analysis of $P$. nigra and $F$. hepatica.

Additional file 2: Host contamination screen for S. japonicum, $F$. hepatica, E. multilocularis and $P$. Xenopodis.

Additional file 3: Fasta sequences of retrieved platyhelminth Dnmt and MBD homologs.

Competing interests

The authors declare that they have no competiting interests.

\section{Authors' contributions}

KKG and KFH conceived the project. NM, JIH, RG, MB, PMB and KB collected or contributed materials for the DNA methylation studies. KKG and IWC carried out the multiple sequence alignments and phylogenetic analyses of 
DNMT and MBD sequences. KKG also performed the MSAP and ELISA analyses of platyhelminth gDNA materials. KKG, IWC and KFH prepared and drafted the manuscript. All authors have read and approved the final manuscript.

\section{Acknowledgements}

This work was supported by an IBERS PhD studentship awarded to KKG and a BBSRC grant (BB/K005448/1) awarded to KFH. We thank Dr. Fred Lewis at the Biomedical Research Laboratory for provision of S. japonicum and S. haematobium parasites (NIH-NIAID schistosome supply contract HHSN272201000005I) and Dr Joseph Jackson (IBERS, Aberystwyth University) for supply of $X$. laevis gDNA. We also thank members of the Hoffmann laboratory (Dr. Emily Peak, Dr. Martha Truscott and Ms Sabrina Munshi), Professor Mike Wilkinson (University of Adelaide), Dr. Carlos M. Rodríguez López (University of Adelaide) and Dr Russell Morphew (IBERS, Aberystwyth University) for many helpful discussions throughout this project. We additionally thank the Parasite Genomics group at the Wellcome Trust Sanger Institute for assistance with gene prediction searches of the $E$. multilocularis, E. granulosus and H. microstomum genomes. Finally, we thank the Developmental Biology and Stem Cell Research group at the Hubrecht Institute and the Helminth Genomics group at Liverpool University for the unpublished Macrostomum lignano and Fasciola hepatica genome sequences used in this study $[64,65]$.

\section{Author details}

${ }^{1}$ Institute of Biological Environmental and Rural Sciences (IBERS), Aberystwyth University, Penglais Campus, Aberystwyth SY23 3FG, United Kingdom. ${ }^{2}$ School of Environmental Science and Development, North-West University, Potchefstroom Campus, Potchefstroom 2520, South Africa. ${ }^{3}$ Institute of Hygiene and Microbiology, University of Würzburg, Würzburg, Germany.

Received: 14 December 2012 Accepted: 3 July 2013

Published: 9 July 2013

\section{References}

1. Park JK, Kim KH, Kang S, Kim W, Eom KS, Littlewood DT: A common origin of complex life cycles in parasitic flatworms: evidence from the complete mitochondrial genome of Microcotyle sebastis (Monogenea: Platyhelminthes). BMC Evol Biol 2007, 7:11.

2. Geyer KK, Hoffmann KF: Epigenetics: A key regulator of platyhelminth developmental biology? Int J Parasitol 2012, 42:221-224.

3. Morison IM, Ramsay JP, Spencer HG: A census of mammalian imprinting. Trends Genet 2005, 21(8):457-465.

4. Altun G, Loring JF, Laurent LC: DNA methylation in embryonic stem cells. J Cell Biochem 2010, 109(1):1-6.

5. Reik W: Stability and flexibility of epigenetic gene regulation in mammalian development. Nature 2007, 447(7143):425-432.

6. Maksakova IA, Mager DL, Reiss D: Keeping active endogenous retrovirallike elements in check: the epigenetic perspective. Cell Mol Life Sci 2008, 65(21):3329-3347

7. Bogdanovic O, Veenstra GJ: DNA methylation and methyl-CpG binding proteins: developmental requirements and function. Chromosoma 2009, 118(5):549-565.

8. Klose RJ, Bird AP: Genomic DNA methylation: the mark and its mediators. Trends Biochem Sci 2006, 31(2):89-97.

9. Cedar $\mathrm{H}$, Bergman $\mathrm{Y}$ : Linking DNA methylation and histone modification: patterns and paradigms. Nat Rev Genet 2009, 10(5):295-304.

10. Fantappie MR, Gimba ER, Rumjanek FD: Lack of DNA methylation in Schistosoma mansoni. Exp Parasitol 2001, 98(3):162-166.

11. Regev A, Lamb ML, Jablonka E: The role of DNA methylation in invertebrates: developmental regulation or genome defense? Mol Bio Evol 1998, 15(7):880-891

12. Raddatz G, Guzzardo PM, Olova N, Fantappie MR, Rampp M, Schaefer M, Reik W, Hannon GJ, Lyko F: Dnmt2-dependent methylomes lack defined DNA methylation patterns. Proc Natl Acad Sci USA 2013. Epub ahead of print.

13. Geyer KK, Rodriguez Lopez CM, Chalmers IW, Munshi SE, Truscott M, Heald J, Wilkinson MJ, Hoffmann KF: Cytosine methylation regulates oviposition in the pathogenic blood fluke Schistosoma mansoni. Nat Commun 2011, 2:424
14. Riutort M, Alvarez-Presas M, Lazaro E, Sola E, Paps J: Evolutionary history of the Tricladida and the Platyhelminthes: an up-to-date phylogenetic and systematic account. Int J Dev Biol 2012, 56(1-3):5-17.

15. Lustigman S, Prichard RK, Gazzinelli A, Grant WN, Boatin BA, McCarthy JS, Basanez MG: A research agenda for helminth diseases of humans: the problem of helminthiases. PLoS Negl Trop Dis 2012, 6(4):e1582.

16. Brehm K: Echinococcus multilocularis as an experimental model in stem cell research and molecular host-parasite interaction. Parasitology 2010, 137(3):537-555.

17. Eisenhoffer GT, Kang H, Sanchez Alvarado A: Molecular analysis of stem cells and their descendants during cell turnover and regeneration in the planarian Schmidtea mediterranea. Cell Stem Cell 2008, 3(3):327-339.

18. Aboobaker AA: Planarian stem cells: a simple paradigm for regeneration. Trends Cell Biol 2011, 21(5):304-311.

19. National Center for Biotechnology Information. http://www.ncbi.nlm.nih.gov/

20. Gasser Lab. http://www.gasserlab.org.

21. Schmidtea mediterranea database. http://smedgd.neuro.utah.edu/.

22. The Taenia solium genome projec. http://www.taeniasolium.unam.mx/taenia/.

23. Schistosoma haematobium database. http://beta.schistodb.net/schisto.beta/.

24. Schistosoma japonicum database. http://www.genedb.org/Homepage/ Sjaponicum

25. Goll MG, Bestor TH: Eukaryotic cytosine methyltransferases. Annu Rev Biochem 2005, 74:481-514

26. Lauster R, Trautner TA, Noyer-Weidner M: Cytosine-specific type II DNA methyltransferases: a conserved enzyme core with variable targetrecognizing domains. J Mol Bio/ 1989, 206(2):305-312.

27. Dong A, Yoder JA, Zhang X, Zhou L, Bestor TH, Cheng X: Structure of human DNMT2, an enigmatic DNA methyltransferase homolog that displays denaturant-resistant binding to DNA. Nucleic Acids Res 2001, 29(2):439-448.

28. Cheng X: Structure and function of DNA methyltransferases. Annu Rev Biophys Biomol Struct 1995, 24:293-318.

29. Pinarbasi E, Elliott J, Hornby DP: Activation of a yeast pseudo DNA methyltransferase by deletion of a single amino acid. J Mol Biol 1996, 257(4):804-813.

30. Goll MG, Kirpekar F, Maggert KA, Yoder JA, Hsieh CL, Zhang X, Golic KG, Jacobsen SE, Bestor TH: Methylation of tRNAAsp by the DNA methyltransferase homolog Dnmt2. Science 2006, 311(5759):395-398.

31. Lavi $T$, Isakov $E$, Harony $H$, Fisher $O$, Siman-Tov R, Ankri S: Sensing DNA methylation in the protozoan parasite Entamoeba histolytica. Mol Microbiol 2006, 62(5):1373-1386.

32. Kuhlmann M, Borisova BE, Kaller M, Larsson P, Stach D, Na J, Eichinger $L$, Lyko F, Ambros V, Soderbom F, et al: Silencing of retrotransposons in Dictyostelium by DNA methylation and RNAi. Nucleic Acids Res 2005, 33(19):6405-6417.

33. Phalke S, Nickel O, Walluscheck D, Hortig F, Onorati MC, Reuter G Retrotransposon silencing and telomere integrity in somatic cells of Drosophila depends on the cytosine-5 methyltransferase DNMT2. Nat Genet 2009, 41(6):696-702.

34. Marhold J, Rothe N, Pauli A, Mund C, Kuehle K, Brueckner B, Lyko F: Conservation of DNA methylation in dipteran insects. Insect Mol Biol 2004, 13(2):117-123

35. Schaefer M, Lyko F: Solving the Dnmt2 enigma. Chromosoma 2010 119(1):35-40.

36. Swain MT, Larkin DM, Caffrey CR, Davies SJ, Loukas A, Skelly PJ, Hoffmann KF: Schistosoma comparative genomics: integrating genome structure, parasite biology and anthelmintic discovery. Trends Parasitol 2011, 27(12):555-564.

37. Hendrich B, Tweedie S: The methyl-CpG binding domain and the evolving role of DNA methylation in animals. Trends in genetics : TIG 2003, 19(5):269-277.

38. Jorgensen HF, Ben-Porath I, Bird AP: Mbd1 is recruited to both methylated and nonmethylated $\mathrm{CpGs}$ via distinct DNA binding domains. $\mathrm{Mol} \mathrm{Cell} \mathrm{BiO}$ 2004, 24(8):3387-3395.

39. Hendrich $\mathrm{B}$, Hardeland $\mathrm{U}, \mathrm{Ng} \mathrm{HH}$, Jiricny J, Bird A: The thymine glycosylase MBD4 can bind to the product of deamination at methylated CpG sites. Nature 1999, 401(6750):301-304.

40. Hendrich B, Bird A: Identification and characterization of a family of mammalian methyl-CpG binding proteins. Mol Cell Biol 1998, 18(11):6538-6547. 
41. Ohki I, Shimotake N, Fujita N, Jee J, Ikegami T, Nakao M, Shirakawa M: Solution structure of the methyl-CpG binding domain of human MBD1 in complex with methylated DNA. Cell 2001, 105(4):487-497.

42. Zou X, Ma W, Solov'yov IA, Chipot C, Schulten K: Recognition of methylated DNA through methyl-CpG binding domain proteins. Nucleic Acids Res 2012, 40(6):2747-2758

43. Ho KL, McNae IW, Schmiedeberg L, Klose RJ, Bird AP, Walkinshaw MD: MeCP2 binding to DNA depends upon hydration at methyl-CpG. Mol Cell 2008, 29(4):525-531.

44. Jiang $C L$, Jin SG, Lee DH, Lan ZJ, Xu X, O'Connor TR, Szabo PE, Mann JR, Cooney AJ, Pfeifer GP: MBD3L1 and MBD3L2, two new proteins homologous to the methyl-CpG-binding proteins MBD2 and MBD3: characterization of MBD3L1 as a testis-specific transcriptional repressor. Genomics 2002, 80(6):621-629.

45. Baubec T, Ivanek R, Lienert F, Schubeler D: Methylation-Dependent and Independent Genomic Targeting Principles of the MBD Protein Family. Cell 2013, 153(2):480-492.

46. Tweedie S, Ng HH, Barlow AL, Turner BM, Hendrich B, Bird A: Vestiges of a DNA methylation system in Drosophila melanogaster? Nat Genet 1999 23(4):389-390.

47. Gutierrez A, Sommer RJ: Functional diversification of the nematode $\mathrm{mbd} 2 / 3$ gene between Pristionchus pacificus and Caenorhabditis elegans. BMC Genet 2007, 8:57.

48. Marhold J, Kramer K, Kremmer E, Lyko F: The Drosophila MBD2/3 protein mediates interactions between the Ml-2 chromatin complex and CpT/Amethylated DNA. Development 2004, 131(24):6033-6039.

49. Wong CP, Magnusson KR, Ho E: Increased inflammatory response in aged mice is associated with age-related zinc deficiency and zinc transporter dysregulation. J Nutr Biochem 2013, 24(1):353-359.

50. Wang $Y$, Jorda M, Jones PL, Maleszka R, Ling X, Robertson HM, Mizzen CA, Peinado MA, Robinson GE: Functional CpG methylation system in a social insect. Science 2006, 314(5799):645-647.

51. Kronforst MR, Gilley DC, Strassmann JE, Queller DC: DNA methylation is widespread across social Hymenoptera. Current biology : CB 2008, 18(7):R287-R288

52. Schaefer M, Lyko F: DNA methylation with a sting: an active DNA methylation system in the honeybee. BioEssays : news and reviews in molecular, cellular and developmental biology 2007, 29(3):208-211.

53. Mandrioli M, Borsatti F: DNA methylation of fly genes and transposons. Cellular and molecular life sciences: CMLS 2006, 63(17):1933-1936.

54. Xiang H, Zhu J, Chen Q, Dai F, Li X, Li M, Zhang H, Zhang G, Li D, Dong Y, et al: Single base-resolution methylome of the silkworm reveals a sparse epigenomic map. Nat Biotechnol 2010, 28(5):516-520.

55. Gao F, Liu X, Wu XP, Wang XL, Gong D, Lu H, Xia Y, Song Y, Wang J, Du J, et al: Differential DNA methylation in discrete developmental stages of the parasitic nematode Trichinella spiralis. Genome Biol 2012, 13(10):R100

56. Lewis FA: Schistosomiasis. In Animal Models for Infectious Diseases, Current Protocols in Immunology. vol. 19. New York: John Wiley \& Sons; 1998:19.1.11-19.1.28. doi:10.1002/0471142735.im1901s28.

57. Tappe D, Brehm K, Frosch M, Blankenburg A, Schrod A, Kaup FJ, MatzRensing K: Echinococcus multilocularis infection of several Old World monkey species in a breeding enclosure. AmJTrop Med Hyg 2007, 77 (3):504-506.

58. Spiliotis M, Brehm K: Axenic in vitro cultivation of Echinococcus multilocularis metacestode vesicles and the generation of primary cell cultures. Methods Mol Biol 2009, 470:245-262.

59. Brehm K, Wolf M, Beland $H$, Kroner A, Frosch M: Analysis of differential gene expression in Echinococcus multilocularis larval stages by means of spliced leader differential display. Int J Parasitol 2003, 33(11):1145-1159.

60. Fernandez C, Gregory WF, Loke P, Maizels RM: Full-length-enriched cDNA libraries from Echinococcus granulosus contain separate populations of oligo-capped and trans-spliced transcripts and a high level of predicted signal peptide sequences. Mol Biochem Parasitol 2002, 122(2):171-180.

61. McManus DP, Knight M, Simpson AJ: Isolation and characterisation of nucleic acids from the hydatid organisms, Echinococcus spp. (Cestoda). Mol Biochem Parasitol 1985, 16(3):251-266.

62. Konrad C, Kroner A, Spiliotis M, Zavala-Gongora R, Brehm K: Identification and molecular characterisation of a gene encoding a member of the insulin receptor family in Echinococcus multilocularis. Int J Parasitol 2003, 33(3):301-312
63. Wellcome Trust Sanger Institute: http://www.sanger.ac.uk/resources/ downloads/helminths/.

64. Macrostomum lignano database. http://www.macgenome.org.

65. Fasciola hepatica database. http://www.liv.ac.uk/genomic-research/.

66. Edgar RC: MUSCLE: multiple sequence alignment with high accuracy and high throughput. Nucleic Acid Research 2004, 32:1792-1797.

67. Punta M, Coggill PC, Eberhardt RY, Mistry J, Tate J, Boursnell C, Pang N, Forslund K, Ceric G, Clements J, et al: The Pfam protein families database. Nucleic Acids Res 2012, 40(Database issue):D290-D301.

68. Hung MS, Karthikeyan N, Huang B, Koo HC, Kiger J, Shen CJ: Drosophila proteins related to vertebrate DNA (5-cytosine) methyltransferases. Proc Natl Acad Sci USA 1999, 96(21):11940-11945

69. Department of Energy Joint Genome Institute. http://genome.jgi.doe.gov/.

70. Fitzpatrick JM, Hirai Y, Hirai H, Hoffmann KF: Schistosome egg production is dependent upon the activities of two developmentally regulated tyrosinases. FASEB J 2007, 21(3):823-835.

71. Huelsenbeck JP, Ronquist F: MRBAYES: Bayesian inference of phylogenetic trees. Bioinformatics 2001, 17(8):754-755.

72. Whelan S, Goldman N: A general empirical model of protein evolution derived from multiple protein families using a maximum-likelihood approach. Mol Biol Evol 2001, 18(5):691-699.

73. Tamura K, Peterson D, Peterson N, Stecher G, Nei M, Kumar S: MEGA5: molecular evolutionary genetics analysis using maximum likelihood, evolutionary distance, and maximum parsimony methods. Mol Biol Evol 2011, 28(10):2731-2739.

74. Figtree v1.3. http://tree.bio.ed.ac.uk/software/figtree/.

75. Bombyx mori database. http://silkworm.genomics.org.cn/.

76. Reyna-Lopez GE, Simpson J, Ruiz-Herrera J: Differences in DNA methylation patterns are detectable during the dimorphic transition of fungi by amplification of restriction polymorphisms. Mol Gen Genet 1997, 253(6):703-710.

doi:10.1186/1471-2164-14-462

Cite this article as: Geyer et al:: Cytosine methylation is a conserved epigenetic feature found throughout the phylum Platyhelminthes. $B M C$ Genomics 2013 14:462.

\section{Submit your next manuscript to BioMed Central and take full advantage of:}

- Convenient online submission

- Thorough peer review

- No space constraints or color figure charges

- Immediate publication on acceptance

- Inclusion in PubMed, CAS, Scopus and Google Scholar

- Research which is freely available for redistribution

Submit your manuscript at www.biomedcentral.com/submit
C BioMed Central 